\title{
Smart Engineering Modeling for Smart Industrial Products
}

\section{László Horváth}

Óbuda University, Doctoral School of Applied Informatics and Applied

Mathematics and John von Neumann Faculty of Informatics

Bécsi út 96/b, H-1034 Budapest, Hungary

horvath.laszlo@nik.uni-obuda.hu

\begin{abstract}
Automation of industrial and consumer products has reached the level on which systems provide for the capability to supervise decisions on physical actions and execution of these actions. Development of these industrial products proceed during their full lifecycle requiring lifecycle integration of innovation, manufacturing, and application as well as utilization the recently emerged methodology of continuous engineering. Engineering in the above level of industrial technology requires contextually integrated model system which serves all research, development, production, and operational activities during the integrated life cycle of product. Smart product requires smart model system which has the capability to recognize situation and apply it at decision on values of relevant product object parameters in virtual to control physical operation of product. This paper introduces recent contributions in modeling for smart engineering. It starts with a novel general model of integrated smart engineering system. Considering this model, smart engineering specific upgrade of formerly published concept of virtual engineering space (VES) is introduced. VES upgrade concentrates on research as key issue in giving smart characteristics for contexts in VES model system. Following this, contextual driving of object parameters is outlined for integrated smart engineering system. The remaining part of paper introduces concept and plan of virtual research laboratory (VRL) in close connection with modeling for integrated smart engineering system. Plans are outlined for next future implementation of the VRL concept and methodology at the Doctoral School of Applied Informatics and Applied Mathematics, Óbuda University. The laboratory system consists of research eligible configuration of the 3DEXPERIENCE engineering modeling platform. It is accessed in the professional cloud of Dassault Systémes.
\end{abstract}

Keywords: system level model of engineering structure; virtual engineering space (VES); contextual driving structure in engineering model; virtual research laboratory (VRL) 


\section{Introduction}

An emerging area in advanced engineering is integrated modeling of engineering structure (ES) for its lifecycle. ES [18] is defined as an industrial or a consumer product, a prototype of a product, or an experimental structure. Lifecycle of ES starts with the first concept and ends with recycling. It covers all ES related engineering activities including conceptualization, research, development, production, marketing, application, user services, and recycling. ES is represented in lifecycle model system [7]. Current leading ES model system is a result of longtime development with emphasis on integration. System level ES uses cooperating systems for control of its operating processes. System level modeling of ES requires a product lifecycle management (PLM) system, which is capable of integrating component models in a contextual model system. In this advanced PLM, purposeful procedures check any component model before it is accommodated in the model system. PLM system which implements the formerly prevailing product data management (PDM) methodology is not suitable for system level ES modeling [8]. When ES is capable of autonomous operation of its physical units using its own decision, we say it is smart. In industrial practice, various other technical and marketing purposed definitions are applied for the relative new term 'smart'.

Modification of a modeling procedure or an entity in model representation in case of smart ES can be executed only in the knowledge of all its consequences in the model system. This request became critical when models started to apply in decisions driving real time physical actions in autonomous smart cyber physical systems (CPSs). Continuous engineering is being developed as methodology to handle the above problem in smart ES operation [2]. The main purpose of continuous engineering is to prevent physical operation malfunctions as a consequence of software modifications which have direct effect on control of those physical operations. In this way, continuous engineering provides a means to avoid erroneous activities of smart cyber control in operation of the CPS physical units. Methodology of Internet of Things (IoT) is highly involved in Continuous engineering. It is obvious that lifecycle development of model system for ES demands methodology from continuous engineering. In this context, authors of [15] define CPS as an approach that targets integration of computational applications with physical devices, relies upon networked and interacting cyber and physical elements, and controls, as well as, monitors real-world physical infrastructures.

Development in the integration of CPS systems places exceptional emphases on behaviors and contexts. Paper [16] introduces the Function-Behavior-State based methodology for integrated CPS design. This methodology applies functional description in multi-domain simulation modeling to represent behavioralstructural aspects of a system. 
Laboratory of Intelligent Engineering Systems, Óbuda University and its predecessor represented three consecutive generations of world level engineering modeling technology during the past two decades. These laboratories applied leading representative engineering modeling systems, which implemented the resource based integrated product information model, the industrial practice driven contextual generic model, and the requirements, functional, logical, and physical (RFLP) structured system-based model [3]. This laboratory was among the first in research and education of the world-famous knowledge ware technology [18]. The experience-based modeling has undergone further development and is currently offered by in the 3DEXPERIENCE platform [19]. Recent, establishment of a fourth laboratory was implemented at the Laboratory of Intelligent Engineering Systems in association with the Doctoral School of Applied Informatics and Applied Mathematics. The Initiative for Model Systems in Engineering of System Based Structures was proposed as the scientific concept for the new laboratory. The 3DEXPERIENCE platform [4] by Dassault Systémes decided to implement as the software system for the new Virtual Research Laboratory (VRL). In this way, conventional organization of modeling capabilities was changed for a platform which offered human role organized apps in cloud. The main activity area of VRL includes modeling of smart systems and realistic simulations, as well as, new areas such as modeling for organic shapes and in bionics.

In this paper, recent research results are introduced as contributions to modeling for smart engineering. Issues in this research include: novel general model of integrated smart engineering system, updated concept of virtual engineering space (VES), contextual driving of objects in smart model system of ES, and concept, plan and implementation of VRL. General model of integrated smart engineering system allows studying requirements against new model structures and representations. Smart engineering specific rethinking of the formerly published concept of virtual engineering space (VES) redefined the utmost objective of model development for ES [5]. Essential requirement against model system for smart ES is an advanced self-adaptive feature combining together the capability for automatic propagation of inside and outside contexts. This is necessary because smart ES is too complex for conventional tracking consequences of frequent modification during its lifecycle.

Concept, methodology, software background, and implementation of a new virtual execution type and experimental model based VRL are also issues in this paper. Cloud environment and research eligible engineering modeling platforms are considered for the new laboratory system. Contrary to many opinions about this style of work, research using experimental model is not only the application of engineering software to assist formerly proven practice but a new style of systematic creative work in a scientifically upgraded modeling environment. VRL is planned to implement relevant achievements discovered in our new century. 
This paper may be difficult to read for those who are not familiar in recent systembased engineering modeling of smart industrial and consumer products. Strong trends towards full contextual integration of innovation, production, and application processes of autonomously operating ES configurations require a changed type of understanding. Huge integrated systems need large amounts of, but integrable contributions. This fact motivated work for contributions in this paper.

\section{General Model of Integrated Smart Engineering System}

The need for representation of all possible contexts in ES model necessitated the definition of the new comprehensive general model of smart engineering system. In this model smart engineering system is considered that which integrates outside driving context sources, lifecycle model system of generic ES, production system for ES, and physically operating ES (Fig. 1).

ES is engineered in an appropriate virtual engineering space (VES) which provides structured description and representation for engineering activities during the lifecycle of ES. Recently, research and development in ES related issues were extended to lifecycle of ES highly relying upon methodology of continuous engineering. To serve this new demand, redefined VES is realized as lifecycle model system of generic ES. VES is developed solely by contributions from outside driving context sources. While the model system of ES is autonomous, it is under continuous control by outside driving contexts for its lifecycle. When it is programmed or considered necessary, intervention by an authorized human is still available at any step of the model development processes. At the same time, an increased level of automation in smart engineering processes restricts human intervention to critical problems. In this way, entities in ES model system are solely under the control of dedicated outside driving contexts. This solution replaces the conventional direct human interaction except for special and often critical cases. The revised VES concept which complies with smart ES and the related smart engineering are discussed in the next section of this paper.

As result of inherent complexity and multidisciplinary nature of systems operated ES lifecycle model system of generic ES demands a wide variety of configurable modeling capabilities during its long-time development. Capabilities are available in role accessible apps as service of engineering modeling platforms. This new era of engineering modeling requires new human thinking and engineering practice which is very different from the former modeling. The lifecycle model system of generic ES must include organized driving background, virtually executable system level model of ES, physical level ES model with realistic simulations, and the capability for smart CPS communication. 
Organized driving background was proposed in papers [6] and [7] among others to establish contextual connections between outside driving context sources and system level model system of ES. This background was organized in driving content structure (DCS) [7]. DCS includes sub-levels in each of its four levels to organize driving contexts for system related aspects. RFLP based ES model [8] is supposed as driven structure. DCS receives, collects, evaluates, and processes contexts from outside driving context sources for lifecycle of ES and provides actual drives for relevant RFLP structure objects. DCS is not an issue in this paper. Above cited publications include all necessary information about it.

The next unit in the general model of integrated smart engineering system is real world operating smart CPS ES. An additional unit here may be the manufacturing system for ES which behaves as CPS considering the recent paradigm, which is mainly defined by Industry 4.x. Driving of manufacturing purposed CPS requires multilevel structured manufacturing model within model system of ES. In [8], manufacturing model structure consists of levels for activities, system, and resources in case of a generic ES.

It is obvious that modeling in this paper must consider physically operating ES and production system for ES as smart CPSs. As it is well known, CPS consists of cyber and physical units where cyber units interact directly with physical units. This interaction is allowed by intelligent sensor networks which always provide actual real-world information about parameters of physical processes. Actuators operate physical processes in accordance with decisions in cyber units. Essentially, cyber units in smart CPS receive and process sensor information about physical unit parameters, recognize situation, and make decision on physical control action in smart CPS in accordance with the recognized situation (Fig. 1). Finally, actuators are controlled in accordance with decision to execute physical actions.

Smart CPS technology is result of long-term development in automatic equipment and device control and in active knowledge representation for engineering model and product operation control. Authors of [14] analyze changes caused by increasingly complex engineered systems. They define smart cyber-physical system, which is empowered by cyber-physical computing features and has capabilities for reasoning, learning, adapting, and evolving.

It is obvious that smart CPS is different from classical CPS. However, this difference, which is often cited as smartness is very difficult to define mainly because only few experiences are available in relevant published theoretical works. In [9], smartness is claimed to derive from cooperative behavior, selfawareness, self-adaptation, and self-optimization. Similar key concepts were analyzed in works published as preliminaries of work in this paper and will be discussed below. It is important to recognize that bioinspired engineering methodology is useful to learn from nature. Findings can be well utilized in development of representations for smart engineering [10]. 


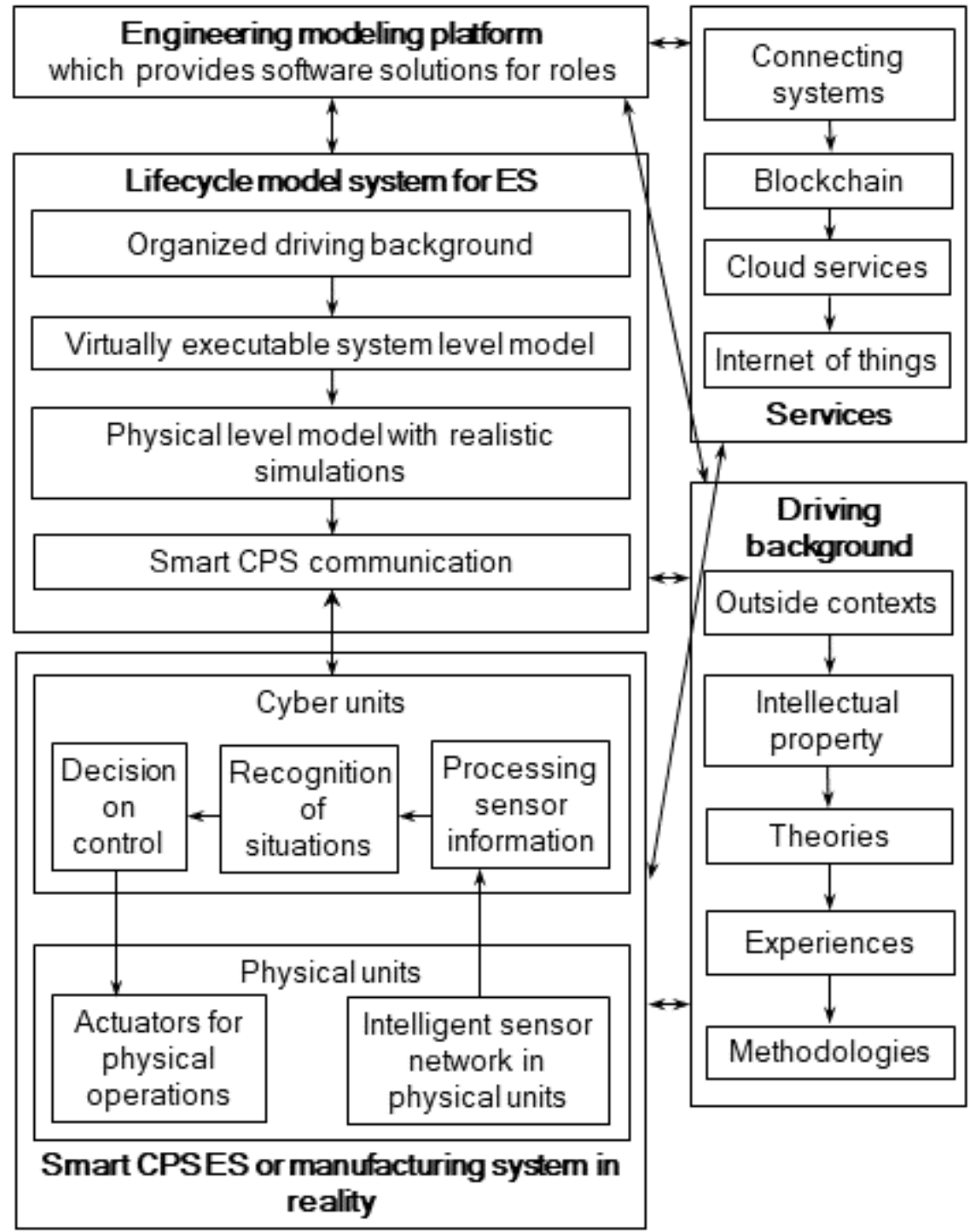

Figure 1

General model of integrated smart engineering system

Importance of behaviors is emphasized in [7] because one of the main purposes of DCS is to drive behavior representations in function (F) and logical (L) level ES components in RFLP structured model system. Behavior representations make F and L level models of ES virtually executable. In case of smart ES, cooperative behavior must ensure mutual understanding between cooperating systems as well as between the system and an intervening human. Cooperative behavior is one of the essential issues in smart CPS because physical activity is generally the result 
of cooperation between systems or systems and humans. With the advent of smart CPS systems erroneous cooperation has become a main cause of malfunctions. A typical source of malfunction in smart CPS is misunderstanding autonomous system decisions and actions by a human who cannot find the proper intervention. Contradictions between situations recognized by different systems are sometimes attempted to be resolved by authorized but inexperienced humans under time pressure. Very rarely, the above problem leads to serious disturbance or crash even in the most advanced aircraft, cars, and industrial processing equipment.

Self-awareness is the capacity of systems to recognize states using analysis of behavior-related situations. This is basically introspection. The situation is applied in decision on model entities or physical unit action. Self-adaptation capability of CPS is also important because inside and outside contexts often change during operation of CPS. As for the ES model system, self-adaption is one of the essential capabilities in leading engineering practice. In this context, selfoptimization proceeds increasingly in real-time and enhances decisions on adaptive changes. Considering the above criteria of smart CPS, smartness is highly based on methodology from intelligent computing, modeling and simulation.

Driving background includes contexts from outside of the model system and CPS as well as from organized intellectual property (IP). Proven and approved theories, experiences and methodologies improve quality of cyber activities and prevent malfunctions in physical units. More details will be given in the Chapter 4 of this paper.

The last block in Fig. 1 shows computing services, which are essential for the operation of smart CPS. In this context, systems are connected, blockchain is managed where it is applied, services often come from cloud, and real time connections between computing and physical devices need availability of advanced IoT services. Computing services are not issues in this paper.

\section{Virtual Engineering Space (VES)}

Virtual engineering space (VES) was published in 2005 and 2007 [11] as a realistic representation of a physically existing or planned engineering space. Today it is more factual than ever before. VES represents a well-defined segment of our physical world where the remained physical world is replaced by contexts in its mutual affect zone. Trusted source ensures valid and realistic context. Sources are responsible for the content of context. At the same time, any relevant and active context is mandatory to include in VES. VES representation must provide valid self-adaptive reaction for context. VES model system can represent human related aspects such as behavior and physiological process. In this paper, VES is supposed to represent an ES. 
Wide availability of cloud-based information technology makes it possible to compose geographically unlimited physical space for a VES. At the same time, recent ES technology demands VES for the representation system based physical space. VES concept was upgraded for systems in [6]. Recently, smart CPS needed new redefinition of VES to better understand this new generation of physical engineering spaces. When implemented, this new VES definition needs engineering modeling platform, which is eligible for the demanded high-level modeling and provides all the capabilities necessary for the representation of VES.

Main structure of smart CPS eligible VES is summarized in Fig. 2. Theoretically, VES consists of its control contexts, model system which represents it, and services. Besides the control contexts, additional VES contexts serve connecting systems and CPS cyber units. Two-way context allows arbitrary direction for its definition. Consequently, connecting system and CPS not only control VES, but they also can be controlled from VES. Dashed boxes include connected outside units which are not issues in this paper.

VES control contexts include authorized human intervention for the definition of entities in VES representation, decisions and measures from higher-level systems such as governments, authorities, company management, standards organizations and research institutions, and proven and accepted intellectual property (IP) from validated sources. Because VES control contexts are continuously changed during the lifecycle of the represented physical space, VES is under continuous change.

VES concept may be applied far beyond usual ESs. Sometimes, VES represents physical space, which has no predictable lifecycle or its lifecycle is considered as unlimited. The following example is for this case. Central buildings of the Óbuda University were built above ruins of the ancient roman city Aquincum. VES representation of an ancient building needs continuous development as new digging experience, archeological research results and other newly emerged contexts are to be included. This is a fantastic new opportunity to bring these ancient buildings to life again. VES here would establish a limited, but progressively evolving realistic self adaptive model, which reacts to any new context by repeated real time simulation. Although the purpose of VES is model based representation, integration and analysis, recent show and additive manufacturing technologies are suitable to visualize VES represented physical objects to assist scientific analysis or support popular demonstration on a higher level than ever before.

Model system collects, validates and connects models. The purpose characterizes projects in which the model system for VES is involved. Structure connects component models. Component model is developed and executed in its own model space considering relevant contexts between models.

Lifecycle model for ES consists of contextual models, which are defined on seven layers (Fig. 2). Level driving communication collects, organizes, validates, and places outside contexts and contexts within the model system. This is followed by 
four levels of RFLP structured model of ES using systems engineering (SE) and latest engineering modeling methodologies.

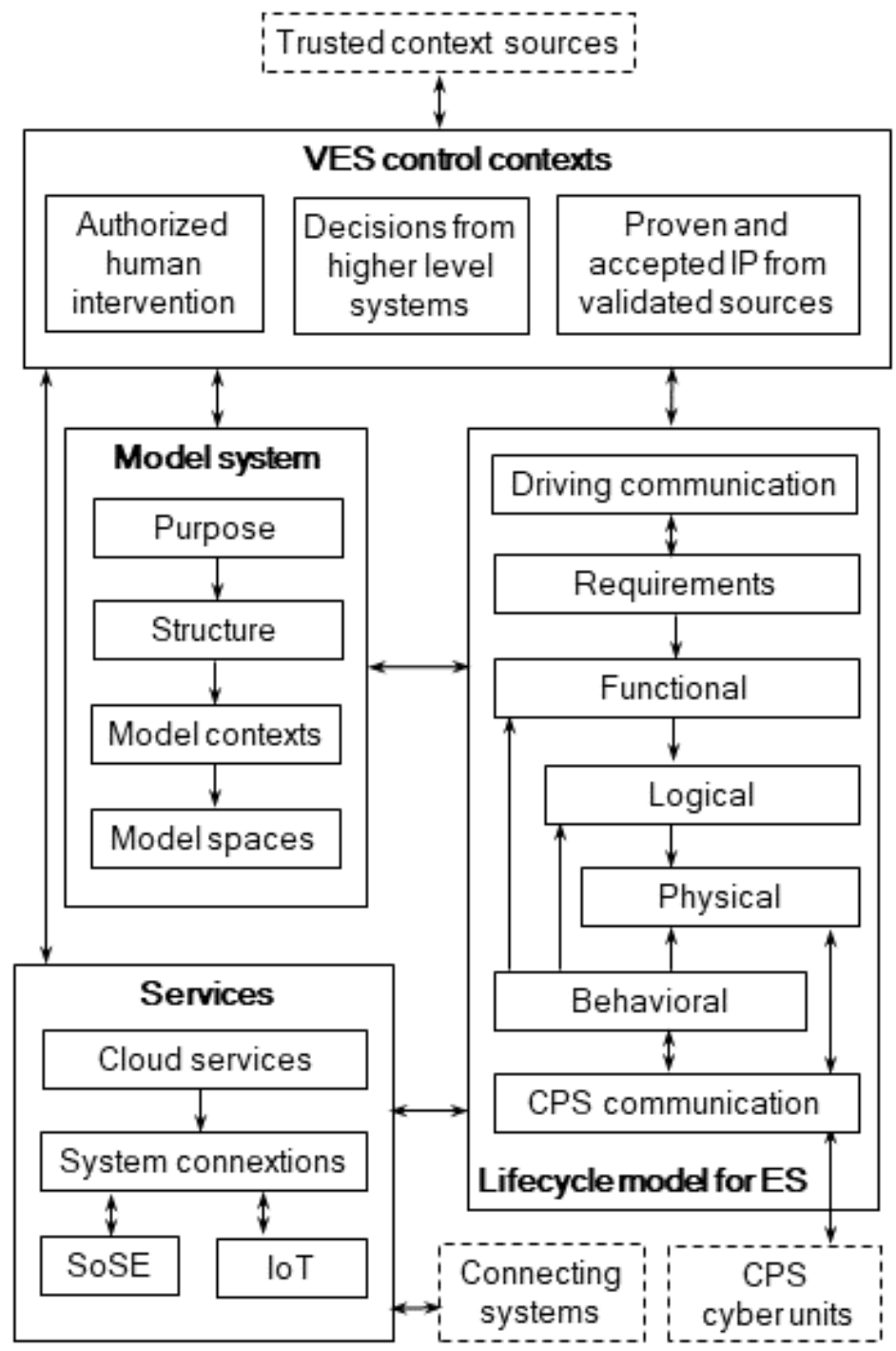

Figure 2

Upgraded VES

The next level is behaviors and serves smart CPS characteristics of VES for situation-based decisions on virtual and physical activities. Finally, seventh level serves bilateral communication with cyber units of CPS systems. In practice, levelling may be different from the above depending on the task and the modeling platform in which the model system is built and managed. 
Services include cloud services, which are essential to provide geographic and time independence of VES definition and application projects, system connections which apply methodology of system of systems engineering (SoSE), and appropriate physical connections using technology from IoT.

\section{Contextual Driving Structure}

The importance of self-adaptive generic engineering model systems was highly increased by wide spreading of industrial and commercial products, which were featured by very complex multidisciplinary structure and smart CPS functions. This main change has brought organized definition of contexts to the foreground. Modern engineering models are capable of high-level and comprehensive representation of contexts between pairs of object parameters in large structures. This achievement makes high-level integration of logical connections, formulas, equations, algorithms, procedures, networks, data arrangements, and stored models possible in model definition. Results from intelligent computing such as Fuzzy, neural networks, evolutionary computing can be integrated in ES model system easily. Theories, methodologies, and experiences are represented in an active model system, which must be driven by consistent system of contexts. Experimental models are developed for well-defined research programs (Fig. 4).

Aspects in contextual driving of model object parameters were published in [6] and [12] including intellectual property (IP) support of model definition and execution. Providing complete and consistent system of contexts is an essential requirement at any time during lifecycle of ES. This is necessary to realize correct context driving of object parameters in the ES model system, which represents smart ES with generic, self-aware, self-adaptation, and self-optimization features.

Current engineering models apply object orientation in an informatically correct manner. Object in a model system is an instance of relevant object class having appropriate parent-children connections in taxonomy. Integration of a new context in a model system supposes that all object parameters connected by the new context are active through appropriate existing contexts.

Essential processing of driving context is introduced in Fig. 3 using a simplified schema to explain the main steps. Context here is supposed as new contribution to context system in an engineering model system. New or modified context must come from well-defined and trusted outside context sources. Parameters which are included in a contextual connection belong to components, elements, features, or other engineering objects represented in ES. Their position in structures and substructures within the model system are defined by special purpose contexts. Owner of any active context takes responsibility during lifecycle of ES. Owner information is included in the model system and available at processing of context. 


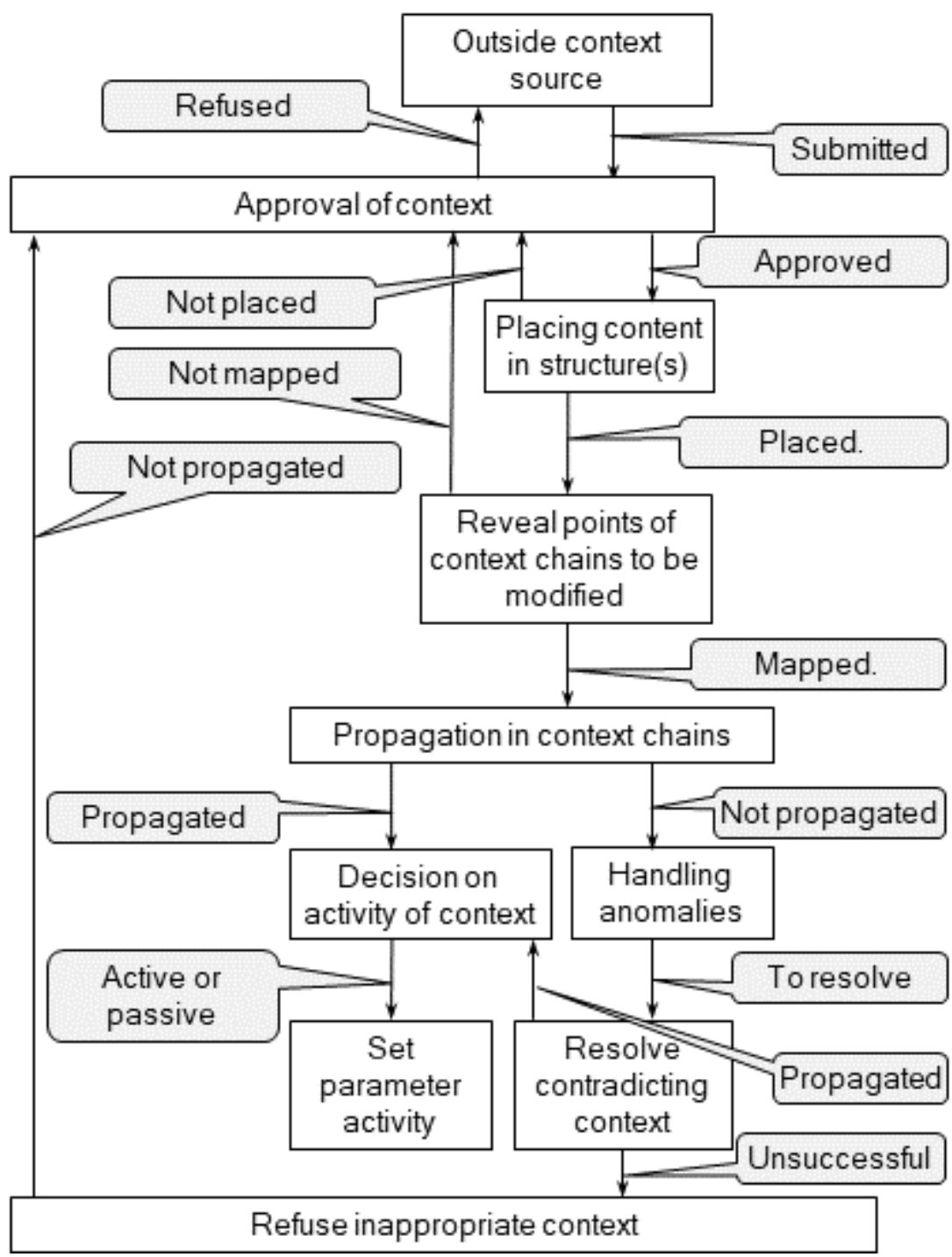

Figure 3

Essential processing of context

Each context has a status parameter, which is changed when a step is completed during its processing. At its submission, context status parameter obtains a submitted value. In the course of the basic approval process, status value changes for approved or refused. Basic approval process decides status considering context parameters such as owner, originality, system relation, discipline, proven principle, method, proven experience, and solution relevance. A former proposal of driving content structure (DCS) included concept for this approval process [7]. 
When a context has approved status, the next step is placing its content in the relevant structure(s). This content will be active in model. It must be suitable for driving the context connected parameter(s) correctly in any situation. Do not forget that the recognized situation, which is applied in decision on real time operation of physical actuators, is critical in correct and safe operation of the relevant smart CPS. Some recently operational problems in advanced smart CPS ESs are often emerged in connection with this issue. Successful placing results placed status of context. Otherwise, this status is unplaced.

The next step in processing of context having placed status is to reveal connecting points in the context chains to be modified. This is an attempt to map the submitted context in relevant content chains of the context system. Successful mapping results mapped status of context. Otherwise, the status in unmapped.

Propagation in context chains means the attempt to execute driving by the new context in the relevant context chains. This requires experiment to propagate context along chains in the context system. When consequences are acceptable, status of context changes for propagated. Otherwise, the new status is not propagated.

When the status is propagated, activity of the new context is set in accordance with its actual position in the model system. In case of not propagated status, attempts for handling anomalies and where necessary resolving context contradiction are done as measures to transform context into valid. When one of these attempts fail, context is refused as inappropriate.

\section{Virtual Research Laboratory (VRL)}

One of the objectives at the implementation of VES is integration of theory and practice in model system of ES. Industry eligible engineering modeling of smart ES was successfully developed towards capabilities, which made parallel representation of theory and relevant experience possible in model system during the past decade. While definition of model object is still based on the latest theory and methodology, theory driven model object generation procedures use representation of relevant valid experience. In this way, experience representation is applied in validation and correction of theory-based parameter values. A milestone was introduction of the knowledge ware in definition and generation of engineering model system to reuse knowledge representing proven best industrial experience and practice in the first decade of the new century. By now, integration of theory and practice has become one of basic criteria in the development of engineering model system.

It is obvious that integrated research, development, manufacturing planning, marketing, application and recycling during the whole life cycle of ES requires 
new ideas and at the same time offers great new possibilities for engineering related research and its laboratory background. In this context, this and the next part of this paper are about concept and implementation of virtual research laboratory (VRL). The term virtual laboratory was applied for laboratories with very different visual and virtual reality related purposes during the past three decades [3]. Some virtual laboratories apply tangible reality to visualize model space and allow interaction between human and this space. Other known purpose of virtual laboratory is extended reality to mix virtual and physical objects to analyze cooperation between them. Additional known purpose of virtual laboratory is to support collaboration amongst capacities for research and associated activities in different geographical places. This collaboration includes shared application of facilities, operation of discipline-specific computing, modeling and simulation software, and sharing collective knowledge and expertise. The models are slightly integrated, and documentation and visualization are still in the center of collaboration activities. The VRL concept is different from the above.

The VRL concept places experimental model and its virtual execution in the center of research activities. VRL requires engineering modeling platform, which is in possession of scientific level functionality suitable for this laboratory purpose. Collaboration between participants of research group is done through experimental model using latest collaboration capabilities available in the modeling platform (Fig. 2). Depending on the research program of VRL, platform is demanded to have capabilities for recently emerged areas such as modeling of systems in ES, organic geometry of shapes and bionics. VRL has the potential to gradually replace most of the physical laboratory functions.

The main activity in VRL is conducting virtual experiments in well-defined research projects with participation from various activity areas, organizations, and geographic sites. VRL activities and models are realized in the host engineering modeling platform. Cloud-based platform host platform provides services for platform management, participant management, role management, and workspace management assuring an environment for virtual experiments and related activities (Fig. 4). Platform capabilities for the representation of deep knowledge are essential for researchers who work in VRL. VRL in the cloud assures highly communicative and distributive research environment.

Virtual experiment process (Fig. 4) is controlled by experiment plan. Starting from an active actual experiment plan, experimental model is initially defined then developed during its whole life cycle considering methodology of continuous engineering. In case of a new experiment, units and entities of experimental model can be newly defined or retrieved as stored object configurations. Stored configurations can be used as building blocks of a new experimental model. It is obvious that experimental model is object model in which instances of object classes are defined in contextual structure. Experimental model is created and managed using modeling capabilities available in the platform including user 
defined resources. When object class, model entity, parameter, procedure, algorithm, function, process, or other new resource required, user definition and configuration capabilities of the platform are applied.

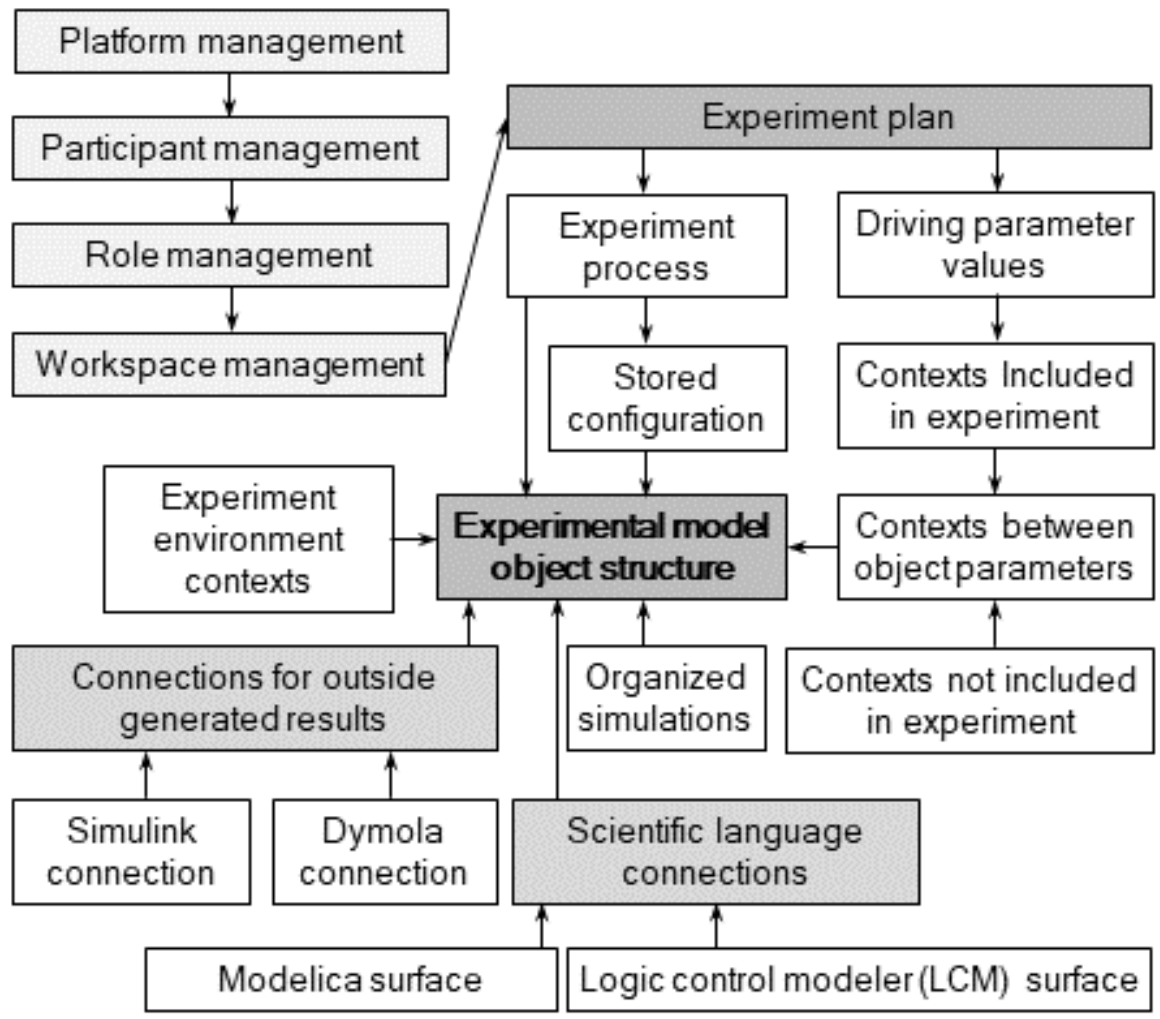

Figure 4

Experiment in VRL

Driving object parameters are analyzed in the course of purposefully defined experiments (Fig. 4). These parameters drive contextually related active objects in the experimental model system. Actual experiments apply relevant experimental model to analyze parameters, their interactions, to find most influential parameters, and to predict parameter values in different situations in accordance with specification in experiment plan. Consistent context system is an essential requirement as in the case of any other model system. All relations of driving parameters and parameters in contextual chains of experimental model must be covered. Using contexts, value sets of driving parameters control execution of experimental model in accordance with actual experiment plan.

Context system of experimental model contains special contexts, which are subjects of analysis and are included in experiment. These contexts carry research 
results, which are to be analyzed and verified in experiments. Objects in experimental model must be suitable to accommodate these contexts. The remaining contexts which are not included in experiments are also active in the experimental model and must serve the relevant experiments. Finally, experiment environment contexts serve connection of experimental model with affect sources in the outside world. These contexts can be used to widen the scope of experiments. Definition and representation of organized simulations serve execution of experimental model in the context structure.

Research often demands software tools which are outside of platform. To solve this problem, 3DEXPERIENCE platform includes software capabilities (apps) to establish communication between the experimental model and the most important outside modeling and simulation software such as Matlab, Simulink, and Dymola. Availability of capabilities which allow accommodation of model representations prepared in Modelica and Logic control modeler (LCM) languages are also important. These languages are inevitable to represent dynamic and state logic behaviors in the experimental model. Owning to features of platform, experimental model can be configured to be integrator in widespread research program. Platform organizes latest complex virtual engineering technology from leading industries so that VRL offers world level solutions for research program. Theory and experience orientations are inherently integrated in models so that contradiction of them, which is usual in conventional engineering can be avoided using appropriately configured and conducted virtual experiments.

Higher education courses which prepare students for roles which are defined in the industrially oriented platform can be reinforced by industrial and research participation. Establishing dual and other cooperation between universities and industrial companies is easy in VRL environment.

\section{VRL in Cloud Organized Platform}

The VRL concept was decided to be implemented mainly for PhD research at the Doctoral School of Applied Informatics and Applied Mathematics, Óbuda University. The Laboratory of Intelligent Engineering Systems is being upgraded for this purpose. Comprehensive capabilities of the 3DEXPERIENCE platform are under installation. Server side is configured in cloud operated by Dassult Systémes as its own system of this VRL. Platform management receives and evaluates inquiries from the local VRL managers as cloud service to define new or changed participant roles and to allocate new apps for participant role. The 3DEXPERIENCE provides a complete cloud package which includes comprehensive modeling software with capabilities in apps (SaaS), platform for custom applications (PaaS), and infrastructure (IaaS). Role provides access to a well-defined set of apps. Research specific roles will be defined for participants. 
Definition and managing custom apps are awaited in VRL research. The Design Apps Developer role is available in the platform for custom definition of apps. This role is important in development of capabilities for representation of reused practice as well as in definition of research program specific methods, procedures, and processes without the need for special programming skill. In this way, research tasks are automated and design assistants are developed. Research will be defined, organized, and conducted in VRL in accordance with its purpose, topic, awaited area, type and publication of results and in coordination with participant institutions, companies and individuals.

Cloud server side of the 3DEXPERIENCE platform of VRL will be accessed by properly installed client workstations. To guarantee the stable operation of the VRL, 3DEXPERIENCE platform certified client workstations will be installed in the next future.

Fig. 5 introduces an example to illustrate that how experiments are planned and conducted. Research for an accredited $\mathrm{PhD}$ topic is supposed to be conducted using the 3DEXPERIENCE based VRL environment at the Doctoral School of Applied Informatics and Applied Mathematics, Óbuda University. This PhD research is expected to produce new representations for the application in model of flexible bodies and function driven organic shapes. The modeled physical system is supposed to include both rigid and flexible elements.

Awaited results of research are divided into theoretical and practical groups. Results in the theoretical group are new algorithms, procedures, object classes, behaviors, situations, networks, rule sets, mathematical functions which modify relevant parameters and contexts in the actual experimental model. Relevant simulations are defined in the experimental model and are conducted using means available within the platform or using one of connected outside solvers. Practical group of research results includes results of execution of the experimental model in the context of appropriate sets of driving parameter values and in accordance with the actual active experiment plan.

The self-adaptive experimental model automatically propagates any modification of contexts then repeats the execution of relevant multiphysics [17] and realistic simulations. Application of continuous engineering methodology is advised to consider in development of experimental models and research results for the full period of a PhD or other actual research. VRL records all important parameters of a research program for the lifecycle of experimental model.

Contexts are revealed and defined for objects inside of the experimental model and for connections of outside objects. Outside affects are handled as contexts defining initial conditions for the experimental program. Among others research task related and higher-level requirements are set here as contexts. Most important outside contexts of the experimental model include value sets for driving input parameters which control experiments and for output of experiments. Practical results are generated by virtual processes. Output parameter values represent performance of results in the theoretical group. 


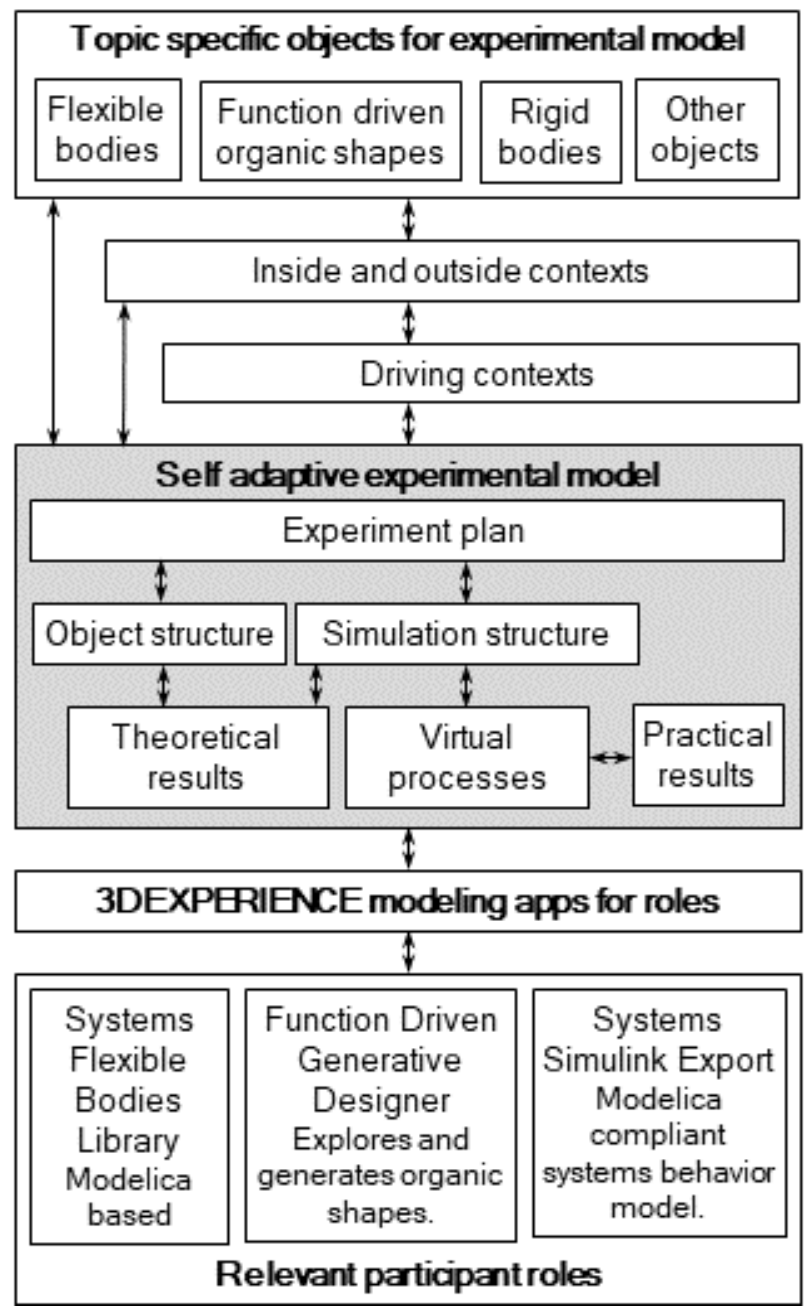

Figure 5

Experiment in VRL

Results of cited research used in a VRL organized research are often available in form which allows their connection to experimental model as context. It is anticipated that this advanced type of citation will gradually replace the conventional passive paper and book citations and at the same time will open the way towards unlimited extension of VRL based research networks in the future. General, cited, and contributed knowledge items are represented as context. General knowledge is available as purchased with the platform. Cited knowledge is owned by other research institutions or individuals. The contributed knowledge is result of research in VRL. The above categories are mandatory to distinguish in all experimental model. 
Student who will work on $\mathrm{PhD}$ thesis for four years in the VRL will be invited as participants in the 3DEXPERIENCE environment of the laboratory. Specific participant roles will serve this special application of platform. Besides VRL specific roles, research will be served by topic dependent 3DEXPERIENCE roles [13]. In the above example research topic (Fig. 5) three roles are included. The Systems Flexible Bodies Library role is based on the Modelica language. The Function Driven Generative Designer role explores and generates organic shapes using functional specification. The Systems Simulink Export role serves Modelica compliant systems behavior models.

\section{Conclusions}

Smart systems operated products, direct connection between model-based virtual decisions and physical actuation, integration of innovation, production and application of ES in a unified cycle and need for reliable lifecycle continuous engineering brought new challenges for physically connected virtual engineering. Above all, smart characteristics of engineering activities will be enforced in the immediate future. Joining in this way of development, this paper introduces some new findings in smart engineering. One of main tasks here is to extend the active context system in generic lifecycle ES model system to outside driving context sources, to production system for ES, and to physically operating ES.

In this paper, contributions to solutions of actual problems of smart engineering include general model of integrated smart engineering system, redefined virtual engineering space (VES) to better understand modeled physical engineering space in case of smart cyber physical system (CPS), an essential processing of new or modified context in consistent context structure, new initiative of engineering model system based virtual research laboratory (VRL), and implementation of VRL concept in cloud organized platform in the Doctoral School of Applied Informatics and Applied Mathematics, Óbuda University.

\section{Acknowledgement}

The author gratefully acknowledges the financial support by the Óbuda University and the Doctoral School of Applied Informatics and Applied Mathematics.

\section{References}

[1] J. Stark: Product Lifecycle Management (Volume 1), $21^{\text {st }}$ Century Paradigm for Product Realisation, Springer, 2015

[2] B. Fitzgerald, K-J. Stol: Continuous software engineering: A roadmap and agenda, Journal of Systems and Software, Vol. 123, 2017, pp. 176-189

[3] L. Horváth: Virtual Research Laboratory for Smart Engineering in the Cloud, proc. of the IEEE $13^{\text {th }}$ International Symposium on Applied Computational Intelligence and Informatics, Timisoara, Romania, 2019, pp. 179-184 
[4] E. Gomez, H. Adli, C. Fernandes, M. Hagege: System Engineering Workbench for Multi-views Systems Methodology with 3DEXPERIENCE Platform. The Aircraft Radar Use Case, Complex Systems Design \& Management Asia, 2016, pp. 269-270

[5] L. Horváth: On the Way Towards Smart Engineering Systems, Proc. of the IEEE $23^{\text {rd }}$ International Conference on Intelligent Engineering Systems, Budapest, Hungary, 2019, pp. 235-240

[6] L. Horváth, I. J. Rudas: Information Content Driven Model for Virtual Engineering Space, Acta Polytechnica Hungarica, Vol. 15, No. 2, 2018, pp. 7-32

[7] L. Horváth, Intelligent Content in System Level Model of Industrial Cyber Physical System, Proc. of the $44^{\text {th }}$ Annual Conference of the IEEE Industrial Electronics Society, Washington D.C., USA, 2018, pp. 29142919

[8] S. Kleiner, C. Kramer: Model Based Design with Systems Engineering Based on RFLP Using V6, Smart Product Engineering, Springer, 2013, pp 93-102

[9] T. Bures, B. Schmerl, D. Weyns, et. al: Software Engineering for Smart Cyber-Physical Systems: Challenges and Promising Solutions, ACM SIGSOFT Software Engineering Notes, 2017, Vol. 42, No. 2, pp. 19-24

[10] L. Horváth: Representing Biological Aspects in Engineering Model System, in Proc. of the IEEE International Work Conference on Bioinspired Intelligence, Budapest, Hungary, 2019, pp. 133-138

[11] L. Horváth: Supporting Lifecycle Management of Product Data by Organized Descriptions and Behavior Definitions of Engineering Objects, Journal of Advanced Computational Intelligence and Intelligent Informatics, Vol. 11, No. 9, 2007, pp. 1107-1113

[12] L. Horváth, Cyber Physical System in Context with System Level Engineering Model, Proceedings of the 2019 IEEE $28^{\text {th }}$ International Symposium on Industrial Electronics, Vancouver, BC, Canada, 2019, pp. $1627-1631$

[13] Dassault Systémes (2019, september 6), 3dexperience portfolio-roles, https://www.3ds.com/products-services/3dexperience/portfolio/

[14] J. Tavčar, I. Horváth, A Review of the Principles of Designing Smart Cyber-Physical Systems for Run-Time Adaptation: Learned Lessons and Open Issues, IEEE Transactions on Systems, Man, and Cybernetics: Systems, Vol. 49, No. 1, 2019, pp. 145-158

[15] P. Leitaoa, A. W. Colomboc, S. Karnouskose, Industrial automation based on cyber-physical systems technologies: Prototype implementations and challenges, Computers in Industry, Vol. 81, 2016, pp. 11-25 
[16] A Canedo, E Schwarzenbach, E. M A Al Faruque, Context-sensitive synthesis of executable functional models of cyber-physical systems, Proc. of the 2013 ACM/IEEE International Conference on Cyber-Physical Systems (ICCPS), Philadelphia, PA, USA, 2013, pp. 99-108

[17] E. L. Blades, R. S. Miskovish, E. A. Luke, E. M. Collins, A. G. Kurkchubashe, A Multiphysics Simulation Capability using the SIMULIA Co-Simulation Engine, Proc. of the $20^{\text {th }}$ AIAA Computational Fluid Dynamics Conference, Honolulu, Hawaii, 2011, pp. 1-16

[18] L. Horváth, J. Fodor, I. J. Rudas, Manufacturing Aspect of the IBCA Structure for Active Knowledge Content Representation in Product Model, IFAC-PapersOnLine, Volume 48, Issue 3, 2015, Elsevier, pp. 1616-1621

[19] Dassault Systémes (2019, september 6), Product Synthesis Solutions, https://www.3ds.com/productsservices/catia/products/v5/portfolio/domain/Product_Synthesis/ 